\title{
Studies of the non-axisymmetric plasma boundary displacement in JET in presence of externally applied magnetic field
}

\author{
D. Yadykin ${ }^{1,2}$, L. Frassinetti ${ }^{3}$, E. Delabie ${ }^{4}$, I.T. Chapman $^{5}$, S. Gerasimov ${ }^{5}$, M. Kempenaars ${ }^{5}$, F. G. \\ . Rimini ${ }^{5}$ and JET Contributors* \\ EUROfusion Consortium, JET, Culham Science Centre, Abingdon, OX14 3DB, UK \\ ${ }^{1}$ Department of Physics and astronomy, Uppsala University, Uppsala, S-75120, Sweden \\ ${ }^{2}$ Department of Earth and Space Sciences, Chalmers University of Technology, Gothenburg, S- \\ 41296, Sweden \\ ${ }^{3}$ Department of Fusion Plasma Physics, Royal Institute of Technology KTH, SE-10044 \\ Stockholm, Sweden \\ ${ }^{4}$ FOM-DIFFER, Nieuwegein, The Netherlands \\ ${ }^{5}$ CCFE, Culham Science Centre, Abingdon, OX14 3DB, UK
}

\begin{abstract}
Non-axisymmetric plasma boundary displacement is caused by the application of the external magnetic field with low toroidal mode number. Such displacement affects edge stability, power load on the first wall and could affect efficiency of the ICRH coupling in ITER. Studies of the displacement are presented for JET tokamak focusing on the interaction between Error Field Correction Coils (EFCCs) and shape control system. First results are shown on the direct measurement of the plasma boundary displacement at different toroidal locations. Both qualitative and quantitative studies of the plasma boundary displacement caused by interaction between EFCCs and shape control system are performed for different toroidal phases of the external field. Axisymmetric plasma boundary displacement caused by the EFCC/shape control system interaction is seen for certain phase values of the external field. The value of axisymmetric plasma boundary displacement caused by interaction can be comparable to the nonaxisymmetric plasma boundary displacement value produced by EFCCs.
\end{abstract}

\section{Section 1. Introduction}

Non-axisymmetric plasma boundary displacement of order of several percent of the minor radius is caused by the application of the external magnetic field with low toroidal mode number. Such displacement was observed in different tokamak devices [1-7] and modelled using 3D equilibrium [8-10] and non-linear stability [11-13] codes. Externally applied fields are used to control or suppress edge localized modes (ELMs) and it is therefore important to understand the effect of the produced plasma displacement on the edge stability. Non-axisymmetric boundary displacement studies are also important for ITER as power heat load on the first wall depends on the plasma boundary position [14] and efficiency of the ICRF coupling depends on the plasma boundary distance to the antenna [15].

* See the Appendix of F. Romanelli et al., Proceedings of the 25th IAEA Fusion Energy Conference 2014, Saint Petersburg, Russia 
Previous studies of the non-axisymmetric plasma boundary displacement in JET were performed using direct measurements either from the reciprocating probe, ECE diagnostic [1] or High Resolution Thomson Scattering diagnostics [2]. A method for plasma boundary reconstruction using magnetic measurements was proposed [2] using direct measurements as scaling factor. It was also observed that the interaction of the Error Field Correction Coils (EFCCs) and shape control system can affect the total displacement but there were no detailed studies of this interaction in JET before.

In this work studies of non-axisymmetric plasma boundary displacement are performed when external magnetic field is applied with toroidal mode number $n=1$. Interaction of the EFCC coils with the shape control system is studied for different configurations of the applied field. The plasma boundary displacement measured by the HRTS and edge charge exchange (ECX) diagnostics is investigated for different phases of the applied external field. It is shown that EFCC/shape control system interaction can substantially affect the total displacement value. It is seen that the plasma boundary displacement evolution differs for different phase of the applied field that is probably caused by the intrinsic axisymmetric component of the EFCC field, as will be shown later. The paper is organized as following: section 2 presents experimental setup showing configuration of the EFCC system, shape controller, and diagnostics used to measure edge plasma boundary displacement. Results investigating interaction of the EFCC system with shape control system and direct measurement of the plasma boundary displacement at different toroidal locations are shown in section 3. The results obtained are discussed in section 4 followed by Conclusions section.

\section{Section 2. Experimental Setup}

Non-axisymmetric plasma boundary displacements are studied when produced by the external magnetic field generated by the set of the of the Error Field Correction coils that are placed in octants 1,3,5,7 outside the first wall. The maximum current in the EFCCs at present is up to $6 \mathrm{kA}$ per turn (multiplied by 16 turns). Signs of the current in the EFCCs can be changed to produce the magnetic field with different toroidal mode number $\mathrm{n}$ and toroidal phase $\phi_{0}$. In this work the magnetic field with toroidal mode number $n=1$ is investigated with different toroidal phase $\phi_{0}$. Signs of the current in the EFCCs set to produce the magnetic field with $\phi_{0}$ values used in this work are given in Table 1.

\begin{tabular}{|c|c|c|c|c|}
\hline & $\phi_{0}=0$ & $\phi_{0}=3 \pi / 4$ & $\phi_{0}=3 \pi / 2$ & $\phi_{0}=7 \pi / 4$ \\
\hline EFCC1 & - & + & 0 & - \\
\hline EFCC3 & 0 & - & + & + \\
\hline EFCC5 & + & - & 0 & + \\
\hline EFCC7 & 0 & + & - & - \\
\hline
\end{tabular}

Table 1. Signs of the EFCC currents to produce the magnetic field mainly with toroidal mode number $n=1$ and different values of the toroidal phase $\phi_{0}$. 
Note that only 2 EFCCs $(1,5$ or 3,7$)$ are active for the configurations used to produce the magnetic field with $\phi_{0}=0,3 \pi / 2$ respectively.

Two diagnostics are used in this work to measure plasma boundary evolution when the nonaxisymmetric external magnetic field is applied: High Resolution Thompson Scattering (HRTS) and Edge Charge Exchange (ECX). HRTS is located in octant $5(\phi=\pi)$ and measures edge electron density and temperature at the outboard side of the torus (just above the midplane). ECX is located in octant 4 (shifted by $\pi / 4$ toroidally with respect to HRTS) and measures edge ion temperature at the outboard side of the torus (measurements are mapped to the midplane). The midplane toroidal row of 8 equally spaced saddle loops [16] measuring the radial magnetic flux is also used in this work for qualitative estimation of the plasma boundary evolution, as it was shown $[2,14]$ that the non-axisymmetric plasma boundary displacement is proportional to the value of main toroidal spectrum component of the radial magnetic field. Sketch of the toroidal cross section of the JET with relative positions of the EFCCs, HRTS, EXC and magnetic diagnostic is shown on Fig. 1

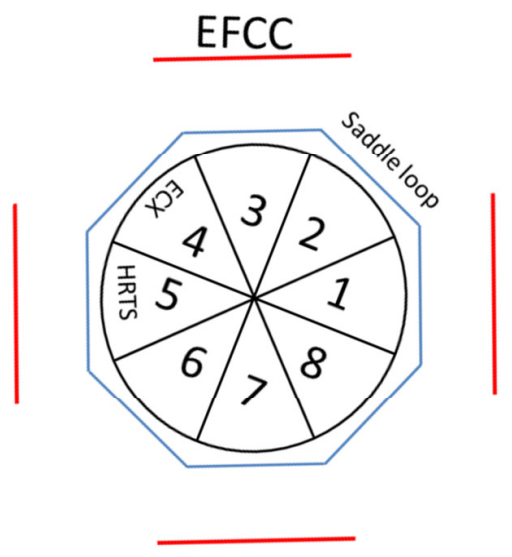

Fig. 1 Sketch showing toroidal JET cross-section together with relative locations of HRTS,ECX, saddle loops and EFCCs, octant numbers marked by numbers.

A shape control system is used in JET in order to control plasma shape and position [17]. Several magnetic diagnostics are used as input to the shape control system. For the present work it is important that part of the input signals (flux measured by saddle loops) are taken mainly from octant 3 (namely 10 saddle loops from octant 3 and 2 saddle loops from octant 7), thus toroidally coincident with the location of one of the EFCCs (see Fig. 1). This fact opens a possibility of interaction between EFCCs and shape control system as saddle loops have direct pick-up of the radial magnetic field generated by EFCCs. Consequences of such interaction will be discussed in the next section.

\section{Section 2. Results}


Results presented in this section are divided into two main parts: studies of the interaction between EFCCs and shape control system; direct measurements of the plasma boundary evolution at different toroidal locations for two phases of the external magnetic field.

\section{Interaction of EFCC and shape control system.}

Interaction between EFCCs and shape control system is possible due to the direct pick-up of the EFCC generated radial magnetic field by the saddle loops that are used as input to the shape control system. This has been studied in detail in MAST (see [18]). Axisymmetric contribution to the total plasma displacement could be produced as response to the non-axisymmetric input. Such effect is potentially dangerous for the plasma operation in JET when using shape controller in GAP mode (i.e. controlling gap(s) between plasma boundary and the first wall) and therefore should be taken into account. Over the years several (empirical) methods were used in order to minimize this effect. One of the methods is to apply preprogrammed midplane outboard gap (ROG control) that follows the shape of EFCC current. Another method (used presently) is to control the inboard midplane gap (RIG control) as the pick-up is much smaller on the inner side of the torus (EFFCs are placed on the outboard side).

The results shown here are obtained when EFCCs were applied to produce the magnetic field with $n=1$ with different toroidal phase $\phi_{0}$ consecutively during one pulse. EFCC current amplitude is changed for each pulse (values in the range 1-5 kA are used). In this way it is convenient to estimate the direct effect from the EFCCs/shape control interaction when using RIG control. Several experimental parameters for the performed experiments are shown on Fig.2. 

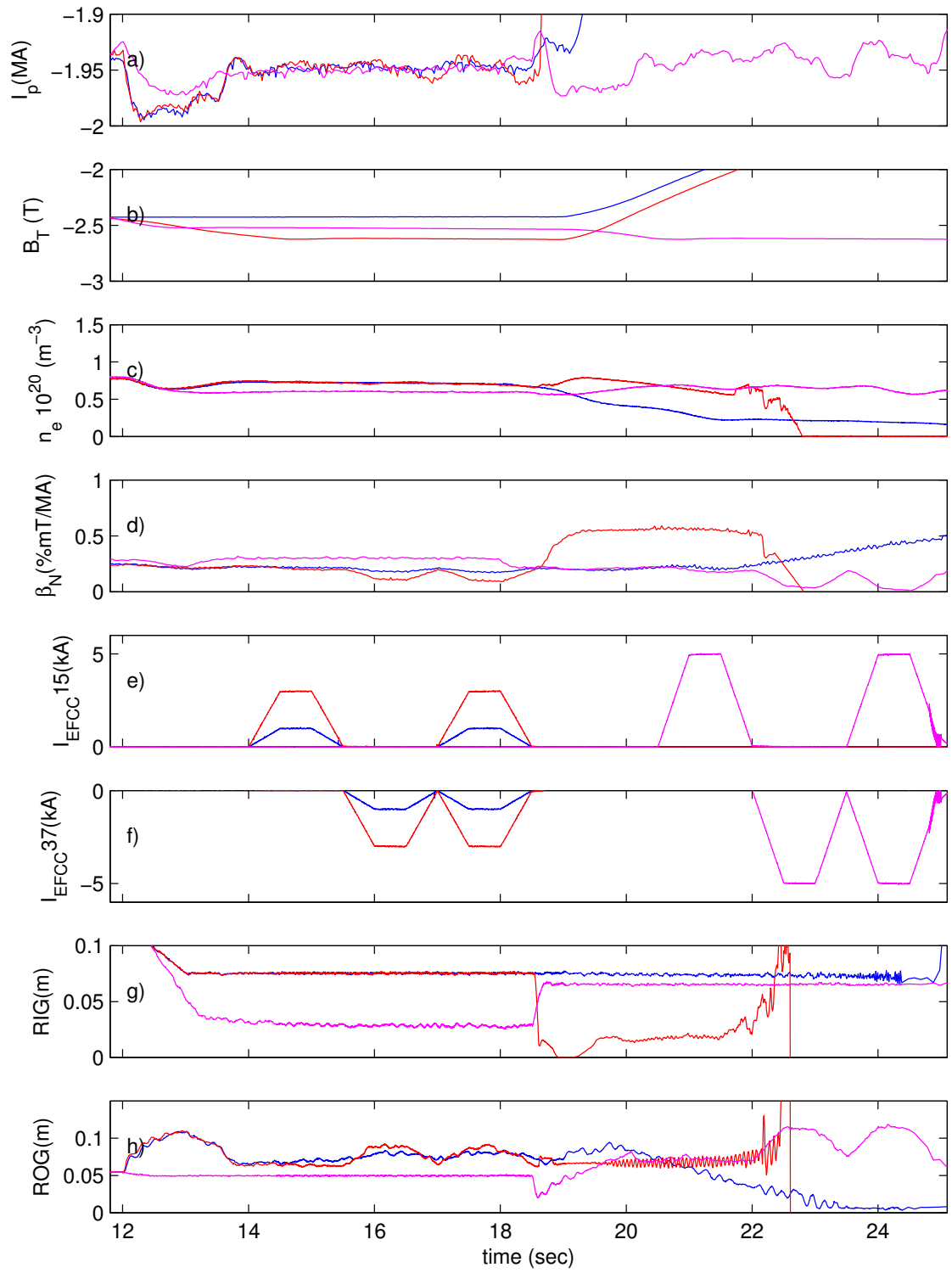

Fig. 2 Experimental parameters for direct EFCC/shape controller studies. Blue - pulse 85676 $\left(I_{E F C C}=1 \mathrm{kA}\right)$; red - pulse $85678\left(I_{E F C C}=3 \mathrm{kA}\right)$, magenta - pulse $85681\left(I_{E F C C}=5 \mathrm{kA}\right)$

L-mode plasmas are used with a low value of the $\beta_{\mathrm{N}}$ parameter $\left(\beta_{\mathrm{N}}=\beta_{\mathrm{t}}\left(\mathrm{aB} \mathrm{B}_{\mathrm{T}} / \mathrm{I}_{\mathrm{p}}\right)\right.$, where $\beta_{\mathrm{t}}=2 \mu_{0}<\mathrm{p}>/ \mathrm{B}_{\mathrm{T}},<\mathrm{p}>$ is the volume average plasma pressure, $\mathrm{B}_{\mathrm{T}}-$ the toroidal magnetic field, $\mathrm{a}-$ the minor plasma radius, $\mathrm{I}_{\mathrm{p}}$ - the plasma current) to avoid possible effects related to the plasma response. Two power supplies are used to feed EFCC coils (same power supply is used for EFCC1, EFCC5 and EFCC3, EFCC7). Time traces of the current amplitudes produced by each 
power supply are plotted on the panels e), f) and marked as $\mathrm{I}_{\mathrm{EFCC}}{ }^{15}, \mathrm{I}_{\mathrm{EFCC}}{ }^{37}$ respectively. In the present studies the current amplitudes produced by the two power supplies are the same (sign of the current at each EFCC determine the phase of the external field, see Table 1). In the following text the current amplitude value will be marked as $\mathrm{I}_{\mathrm{EFCC}}$ without referring to the particular power supply. The following phases of the external field are applied consecutively for each value of EFCC current with $\Delta \mathrm{t}=1.5 \mathrm{sec}$ interval: 1) $\phi_{0}=0$; 2) $\phi_{0}=3 \pi / 2$; 3) $\phi_{0}=7 \pi / 4$. Note that the start time of EFCC is shifted for the case of $\mathrm{I}_{\mathrm{EFCC}}=5 \mathrm{kA}$. The shape control system is set to the gap control mode with outer gap control (ROG) up to $12 \mathrm{sec}\left(19 \mathrm{sec}\right.$ for case $\left.\mathrm{I}_{\mathrm{EFCC}}=5 \mathrm{kA}\right)$, switched to the inner gap control (RIG) afterwards.

A toroidal row of 8 equally spaced saddle loops is used, located at the outboard side of the torus (just above the midplane poloidally) in order to study the pick-up effect. Toroidal spectral decomposition of the measured radial magnetic flux is performed to estimate the pick-up effect. Evolution of the amplitude of resulting toroidal spectral components is shown on Fig.3.

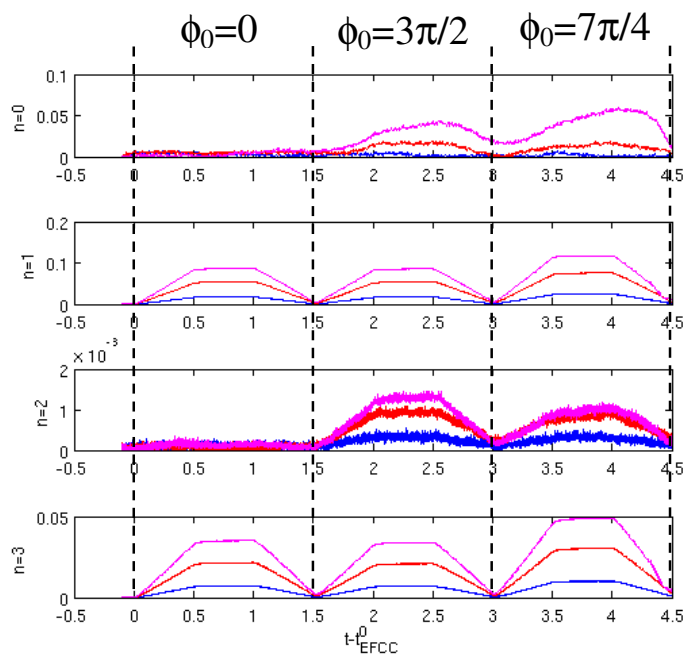

Fig. 3. Toroidal spectrum of radial magnetic flux measured by the outboard row of the saddle loops. Blue - pulse $85676\left(I_{E F C C}=1 \mathrm{kA}\right)$; red - pulse $85678\left(I_{E F C C}=3 \mathrm{kA}\right)$, magenta - pulse 85681 $\left(I_{E F C C}=5 \mathrm{kA}\right)$. The time vector is shifted to have zero at the time of the EFCC initial switching on $\left(t_{E F C C}^{0}\right)$.Vertical dashed lines mark time intervals when different phases of the external filed are applied (phase values are shown above the panels)

The time base is shifted to have zero at the start time of EFCC for all phase values. This is done in order to remove the time shift for the case $\mathrm{I}_{\mathrm{EFCC}}=5 \mathrm{kA}$ (see Fig.2). An axisymmetric component $(\mathrm{n}=0)$ should be zero in general (as an $n=1$ non-axisymmetric current is produced by EFCCs). It is seen in contrary that the $n=0$ component is not zero when EFCCs are applied with $\phi_{0}=3 \pi / 2$, $\phi_{0}=7 \pi / 4\left(\mathrm{t}-\mathrm{t}^{0}{ }_{\mathrm{EFCC}} \geq 1.5 \mathrm{sec}\right)$. The time behavior of $\mathrm{n}=0$ component follows that of EFCC current (see $n=1$ component trace) suggesting that $n=0$ component is produced due to the interaction between the EFCCs and the source of axisymmetric field. Comparison of the configurations with 
$\phi_{0}=0$ and $3 \pi / 2$ shows that this source is the shape control system. In these configurations $n=1$ field is produced by one pair of the EFCCs (either 1,5 or 3,7, see Table 1) with $\left|\Delta \phi_{0}\right|=\pi / 2$ phase shift between configurations. It is seen that the amplitude of $n=0$ component is almost zero for the case $\phi_{0}=0\left(\mathrm{t}-\mathrm{t}^{0}{ }_{\mathrm{EFCC}}<1.5 \mathrm{sec}\right)$. This is due to the fact that when $\mathrm{n}=1$ field is created by only one pair of EFCCs the magnetic field measured by the sensors coincident with another pair is zero $\left(\pi / 2\right.$ phase difference). In our case when EFCCs 1,5 are applied $\left(\phi_{0}=0\right)$ the field measured by the sensors in octants 3,7 is zero and no pick-up is translated to the shape control system (current in EFCCs 3,7 is zero). The situation is different when a configuration with $\phi_{0}=3 \pi / 2$ (EFCCs 3,7 are active) is applied. Direct pick-up of the field produced by EFCCs is translated to the shape control system thus creating an axisymmetric component. Direct pick-up is also seen when the external field is applied with the phase value $\phi_{0}=7 \pi / 4$ (as EFCCs 3,7 are active for this case).

It is seen that harmonics with higher toroidal numbers $(n=2,3)$ are present in the spectrum (Fig 3). This can be attributed to so-called side band effect appearing due to the discrete nature of EFCCs $[19,20]$. Interesting to note that periodicity 2 (i.e. sideband harmonics with $n_{\text {s.b }}=n_{0}+i^{*} 2$, where $\mathrm{n}_{\mathrm{s} . \mathrm{b}}$ is the toroidal number of side band, $\mathrm{n}_{0}$ is the toroidal number of main harmonic,in our case $\mathrm{n}_{0}=1$, and $\left.\mathrm{i}= \pm 1, \pm 2, \pm 3 \ldots\right)$ is seen for all EFCC configurations. This is natural for configurations with $\phi_{0}=0,3 \pi / 2$ where 2 coils are active but not obvious for configuration with $\phi_{0}=7 \pi / 4$ where all coils are applied (expected periodicity in this case is 4 ).

\section{Direct measurements of the plasma boundary evolution at different toroidal positions.}

In order to study non-axisymmetric plasma boundary evolution it is important to have direct measurements of the plasma boundary displacement at different toroidal locations. Several diagnostics able to track plasma edge evolution exist at JET. Two such diagnostics are used in this work: High resolution Tomson Scattering (HRTS) measuring plasma density profile and edge charge exchange (ECX) diagnostics measuring ion temperature at the plasma edge. Diagnostics are shifted by $\pi / 4$ toroidally (see Section 2). The measurements are taken in H-mode for two values of toroidal phase of the external magnetic field $\left(\phi_{0}=3 \pi / 4\right.$ and $\left.\phi_{0}=7 \pi / 4\right)$. Time traces of several experimental parameters for the performed experiments are shown on Fig. 4. 

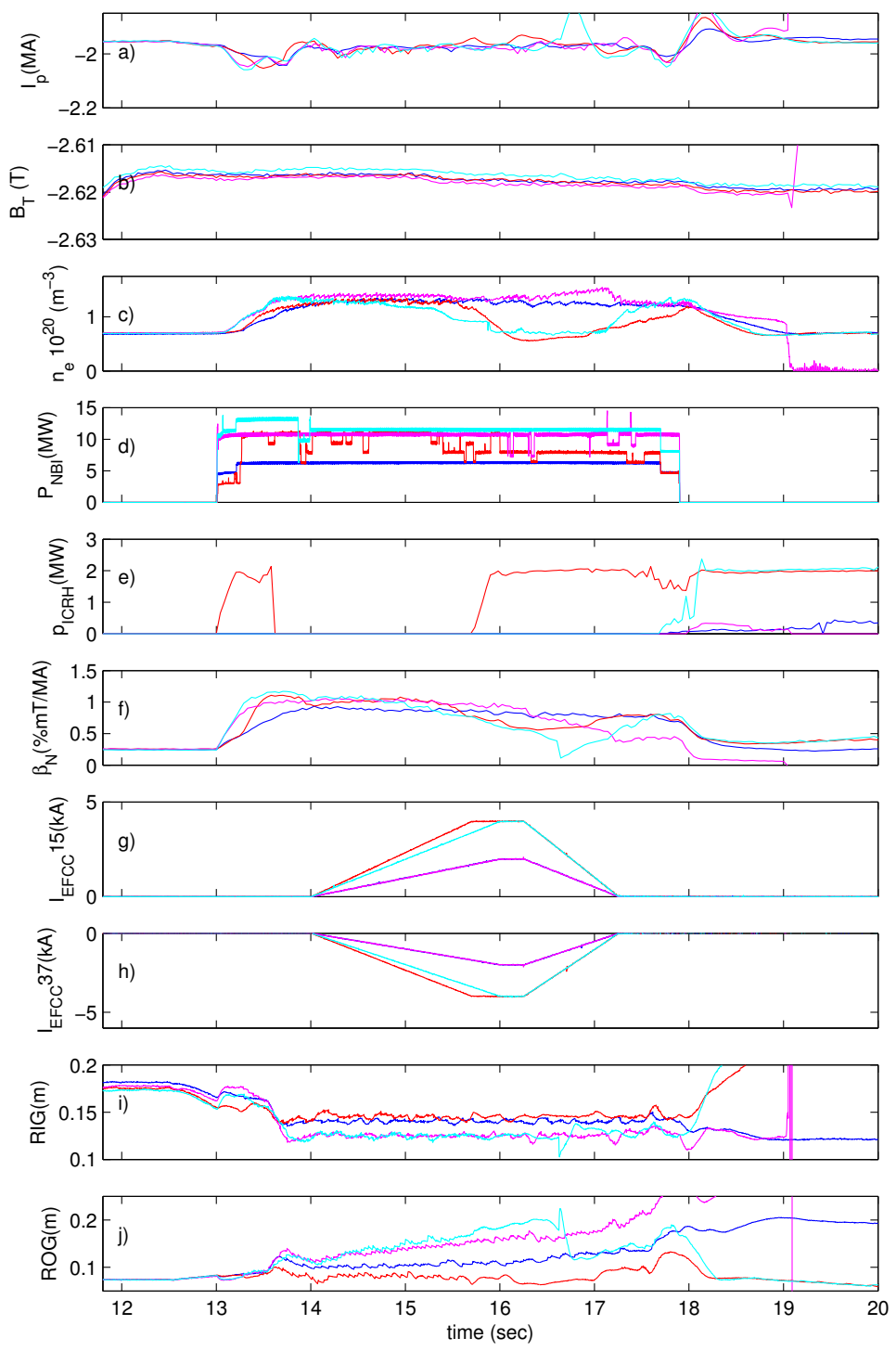

Fig. 4. Experimental parameters for studies of the plasma boundary displacement for the two toroidal phase values of the external magnetic field. Blue - pulse $87041\left(\phi_{0}=3 \pi / 4, I_{E F C C}=2 \mathrm{kA}\right)$; red - pulse $87044\left(\phi_{0}=3 \pi / 4, I_{E F C C}=4 k A\right)$; magenta - pulse $86929\left(\phi_{0}=7 \pi / 4, I_{E F C C}=2 \mathrm{kA}\right) ;$ cyan pulse $87118\left(\phi_{0}=7 \pi / 4 \mathrm{deg}, I_{E F C C}=4 \mathrm{kA}\right)$. 
EFCC currents with different amplitudes $\left(\mathrm{I}_{\mathrm{EFCC}}=2 \mathrm{kA}, \mathrm{I}_{\mathrm{EFCC}}=4 \mathrm{kA}\right)$ are applied for each toroidal phase value. In all experiments internal gap control (RIG) is applied meaning that interaction between shape controller and EFCCs can affect the results.

Evolution of the plasma boundary measured by HRTS and ECX diagnostics for the case $\phi_{0}=3 \pi / 4$, $\mathrm{I}_{\mathrm{EFCC}}=2 \mathrm{kA}$ is shown on Fig. 5. Plasma density profile measured by HRTS is fitted at the edge [21] and the radial position of the middle of the pedestal is followed to get evolution of the plasma boundary at the toroidal position $\phi=\pi$. In order to get boundary position from ECX measurements (toroidal angle value $\phi=3 \pi / 4$ ), intensity radial profile is used (instead of post processed ion temperature measurements) and specific (threshold) value of intensity is used to mark the boundary position.
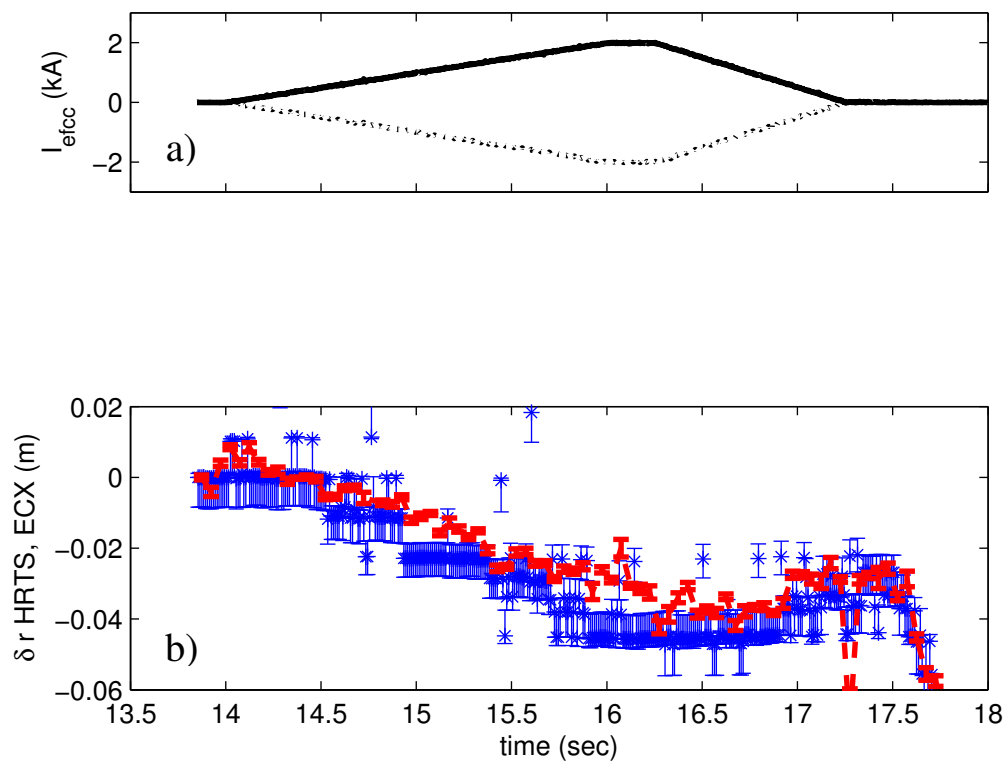

Fig. 5. Plasma boundary displacement measured by HRTS (octant 5) and ECX (octant 4) diagnostics for the case $I_{E F C C}=2 k A, \phi_{0}=3 \pi / 4$. a) $I_{E F C C}$, solid - EFCC15, dotted-EFCC37; b) plasma boundary displacement, red (dashed)- HRTS, blue (stars) - ECX.

Error bars for ECX measurements represent the distance to the next radial channel of the diagnostics, the error bars for HRTS diagnostic is from the uncertainty in the mtanh (modified hyperbolic tangent function) fitting parameters. (see [21] for details). It is seen that the results are in agreement in general. The maximum displacement seen for both diagnostics is around $4 \mathrm{~cm}$ (negative value means inward displacement). It should be noted that total displacement seen can be caused not only by the direct non-axisymmetric field generated by EFCCs. This was, for example, the case for the previous studies [2] where reference pulse (same scenario, no EFCCs) was used to remove all other contributions. Although it worked satisfactorily there, the results shown in this work suggest that some contributions (for example axisymmetric plasma boundary 
displacement caused by the interaction between EFCCs and shape controller) can not be removed using reference pulse. Moreover, no reference pulse was performed for these experiments.

In order to understand the nature of the observed displacements it is useful to compare a toroidal map of the applied external field (main source of the observed non-axisymmetric displacements) with the kinetic measurements [3]. It was mentioned earlier (see Fig. 3) that the toroidal spectrum of the applied external perturbation is not clean, i.e. side band harmonics are present apart from the main harmonic $(n=1)$. The vacuum toroidal structure for the two phase values of the external field is shown on Fig. 6 measured by the outboard row of the magnetic saddle loops.

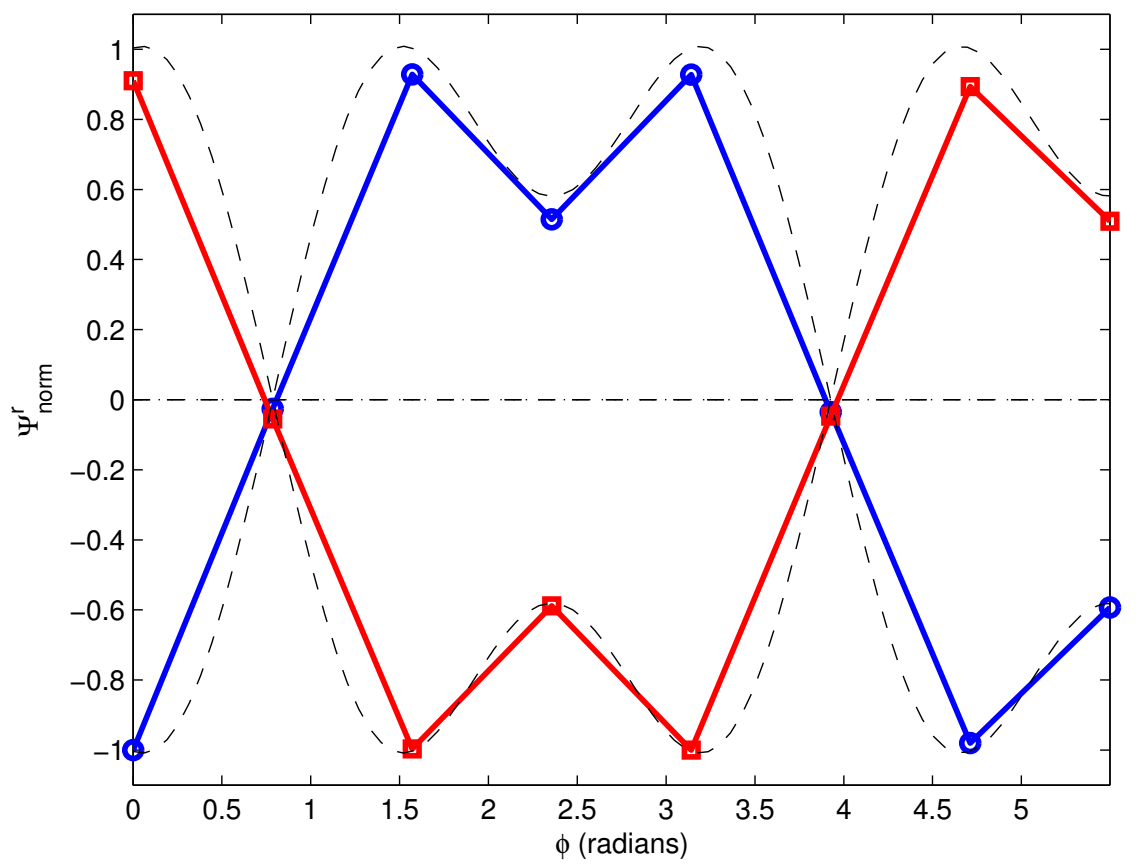

Fig. 6. Vacuum toroidal structure of the external field. Solid lines - measured by the outboard row of the saddle loops, symbols point measurement locations for the field phase values $\phi_{0}=3 \pi / 4$ (squares) and $\phi_{0}=7 \pi / 4$ (circles). Dashed lines - analytical approximation

$$
\psi_{\text {anal }}=0.42 * \sin \left(3 \phi+\phi_{0}\right)+\sin \left(\phi+\phi_{0}\right)
$$

The analytical approximation of the measured structure is also shown here assuming $n=1$ and $n=3$ spectral components to be the content of the total perturbation. It is seen that the analytical approximation agrees well with measured structure. Comparison of the toroidal structure of the externally applied field and the perturbation of the plasma edge measured by HRTS and ECX diagnostics is shown on fig. 7 . 


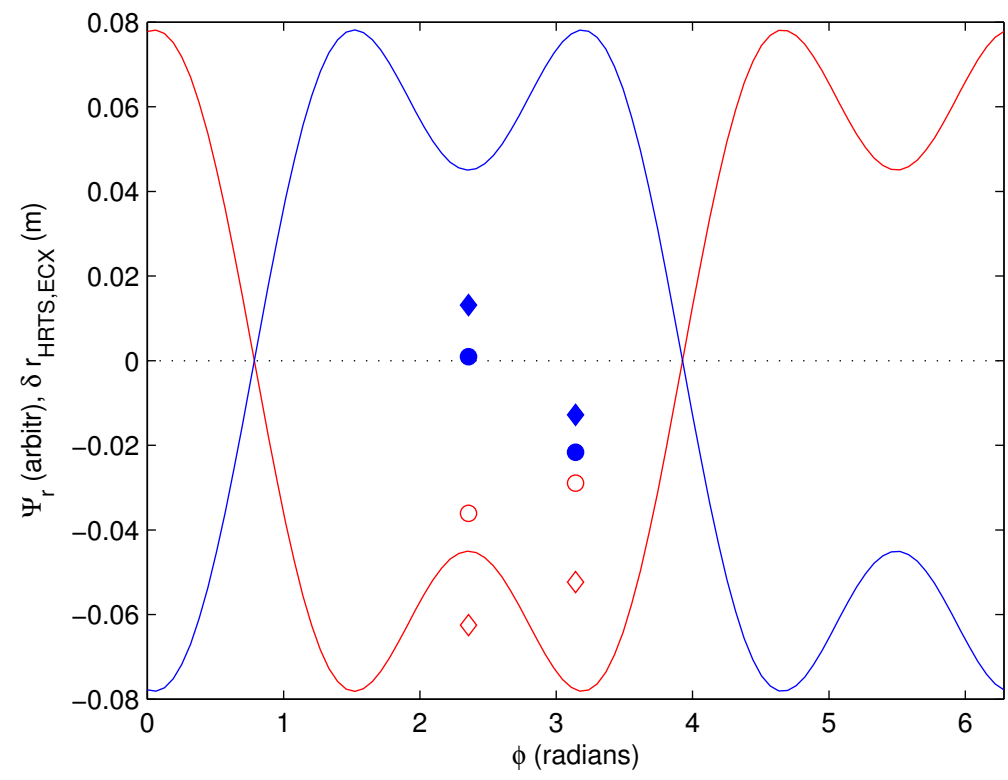

Fig. 7. Comparison of the plasma boundary displacement measured by HRTS and ECX (symbols) and toroidal structure of the external filed (solid lines). Measurements are taken for two phases of the external field ( $\phi_{0}=3 \pi / 4 \mathrm{deg}$, open symbols, $\phi_{0}=7 \pi / 4 \mathrm{deg}$, filled symbols) and two amplitudes of the EFCC currents $\left(I_{E F C C}=2 \mathrm{kA}\right.$-circles, $I_{E F C C}=4 \mathrm{kA}-$ diamonds $)$. Amplitude of the magnetic flux shown is arbitrary.

It is seen that measured displacement depends both on the toroidal angle and on the amplitude of the applied field (circles vs diamonds). Relative dependence on toroidal angle and on the amplitude of EFCC current is approximately preserved for the one phase value of the external field (open or closed symbols), i.e. $\delta \mathrm{r}\left(\mathrm{I}_{\mathrm{EFCC}}\right)$ is the same for different toroidal angles, similarly $\delta \mathrm{r}(\phi)$ is the same for different $\mathrm{I}_{\mathrm{EFCC}}$. But these relative dependencies are different for different phase values. Also no obvious qualitative agreement is seen between vacuum structure of the applied field and the observed plasma boundary displacement (solid curves vs symbols). This will be discussed more below.

\section{Section 4. Discussion}

Studies of the JET plasma boundary displacement in presence of the externally applied magnetic perturbations are performed in this work. The results obtained show that: a) interaction of EFCCs and shape control system can cause additional axisymmetric plasma boundary distortion for certain EFCC configurations; b) non-axisymmetric plasma boundary displacement measured at different toroidal locations depends on the phase and amplitude of the externally applied magnetic field. In order to understand source of the observed displacement it is useful to investigate spectral content of the total magnetic field. It was mentioned several times above that the total magnetic field observed contains several toroidal spectral harmonics. Toroidal structure 
of the total vacuum magnetic field with the phase value $\phi_{0}=3 \pi / 4$ is shown on Fig. 8 together with the structure of the separate toroidal components with $n=1$ and $n=3$.

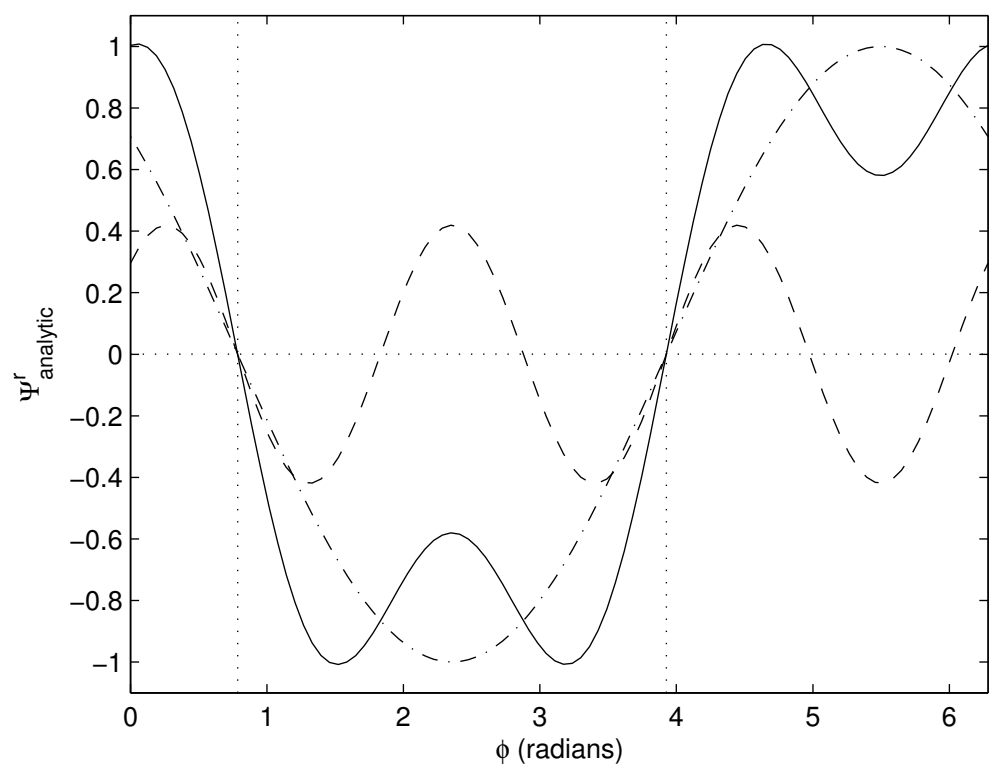

Fig. 8. Toroidal structure of the spectral content of the vacuum magnetic field with $\phi_{0}=3 \pi / 4$. Solid - total field, dashed-dotted $-n=1$ component, dashed $-n=3$ component

Comparison of the observed displacement with particular spectral components of the vacuum field is shown on Fig. 9. It is seen that the observed displacement agrees well with the shape of the $n=1$ spectral component for both phase values of the applied field (agreement is better for both values of the field amplitude for the case $\phi_{0}=3 \pi / 4$ ). 

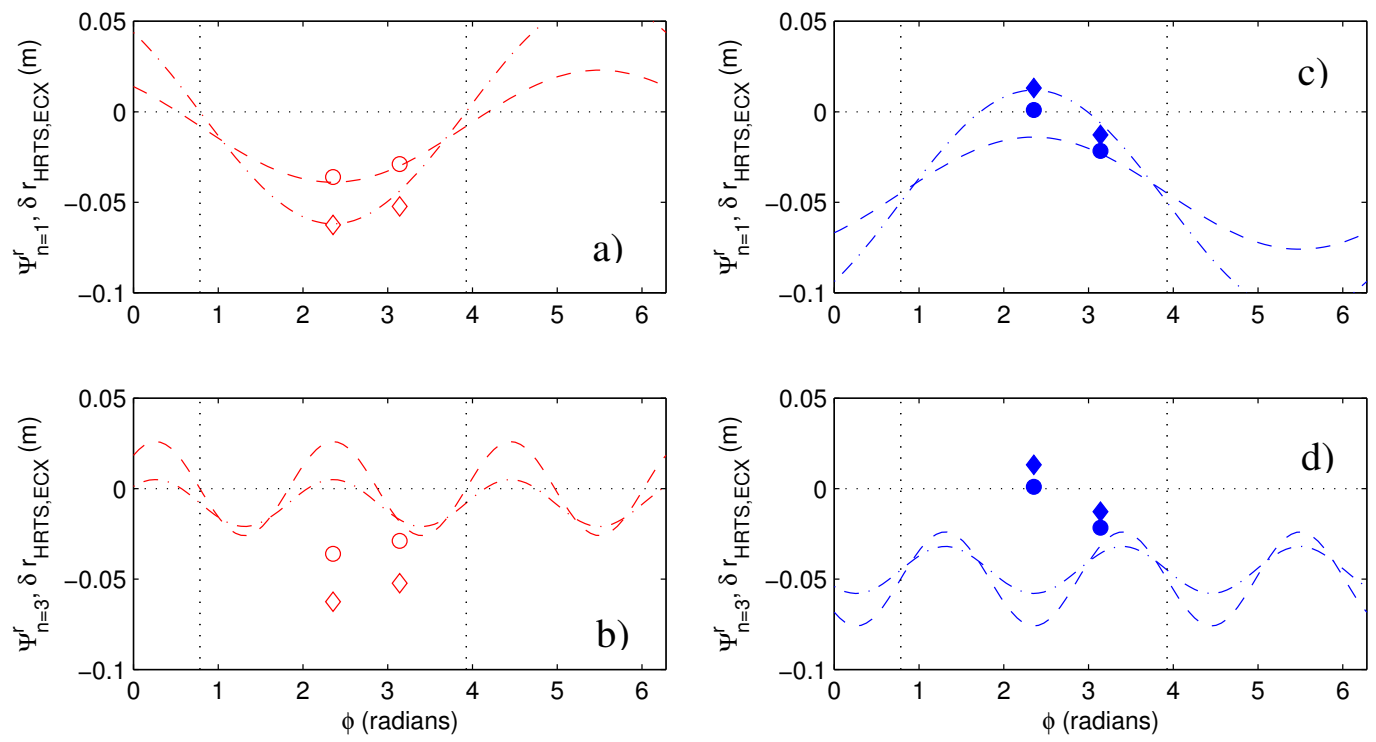

Fig. 9. Comparison of the measured plasma boundary displacement with toroidal components of the vacuum magnetic field. a) $\left.\phi_{0}=3 \pi / 4, n=1, b\right) \phi_{0}=3 \pi / 4, n=3$, c) $\left.\phi_{0}=7 \pi / 4, n=1, d\right) \phi_{0}=7 \pi / 4, n=3$.

Dashed line $-I_{E F C C}=2 k A$, dashed-dotted line $-I_{E F C C}=4 \mathrm{kA}$. Absolute amplitude of the field components is arbitrary, relative amplitudes are consistent (i.e. the amplitude for the case $I_{E F C C}=2 \mathrm{kA}$ is half of the amplitude for the case $I_{E F C C}=4 \mathrm{kA}$ ).

An axisymmetric component (identified by the nonzero field values at the toroidal positions marked by the vertical dotted lines, see also Fig. 8) has been added for the case $\phi_{0}=7 \pi / 4$ in order to match the measurements. Comparison of the toroidal structure of the plasma magnetic flux measured for the two values of $\phi_{0}$ is performed in order to understand presence the of $n=0$ displacement. Results of the comparison are shown on Fig. 10 for several time points. 

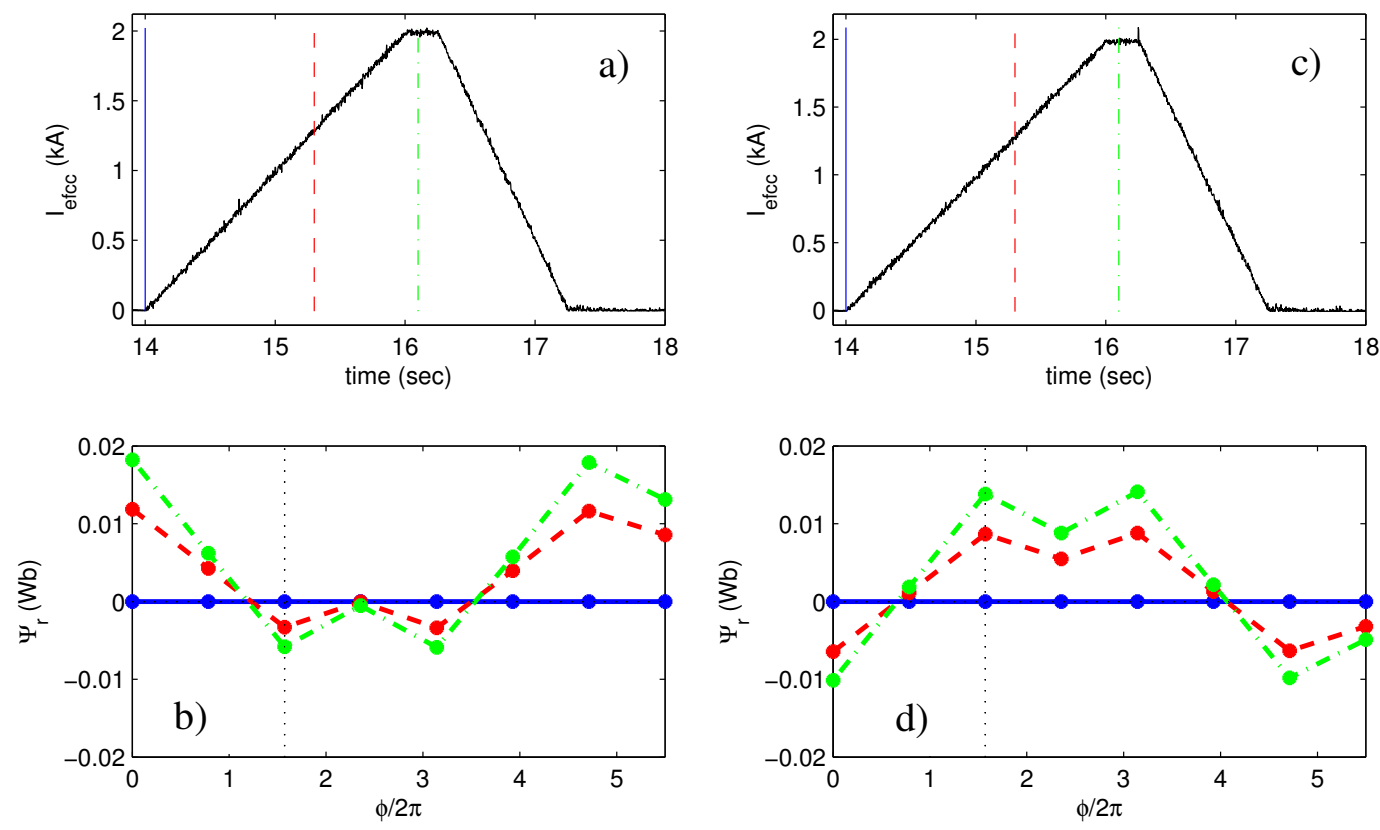

Fig. 10. Toroidal structure of the total plasma radial flux measured by the row of outboard saddle flux loops. a) time evolution of the EFCC current for the case $\left.\phi_{0}=3 \pi / 4 ; b\right)$ toroidal structure of the total radial flux for the case $\phi_{0}=3 \pi / 4$ measured at several time points; $c$ ) time evolution of the EFCC current for the case $\phi_{0}=7 \pi / 4 ; d$ ) toroidal structure of the total radial flux for the case $\phi_{0}=7 \pi / 4$ measured at several time points. Vertical lines on the panels a),c) show time points where measurements of the flux are taken (line styles are consistent with those on the panels $b$ ), $d$ ) ). Verical dotted lines on panels $b$ ), $d$ ) mark toroidal position where magnetic diagnostics are located used as input to the shape control system.

It is seen that the total flux for the case $\phi_{0}=3 \pi / 4$ contains 'intrinsic' $n=0$ component that acts as to decrease magnetic flux at the toroidal position where diagnostic measurements are taken for the shape control system (marked by the dotted vertical line), thus decreasing effect from the interaction of the EFCCs and the shape control system. Further studies are needed in order to confirm the results. It is also not clear for now what causes the 'intrinsic' axisymmetric component observed.

Based on the results obtained it is now possible to estimate the plasma boundary displacement caused by the interaction of the EFCC coils and shape control system. Assuming no effect from the interaction for the case $\phi_{0}=3 \pi / 4$ (see fig. 9) and the same non-axisymmetric component amplitude for both phases of the external field, the axisymmetric displacement for the case $\phi_{0}=7 \pi / 4$ is $\delta \mathrm{r} \sim 3 \mathrm{~cm}$ for the $\mathrm{I}_{\mathrm{EFCC}}=2 \mathrm{kA}$ that is actually close to the non-axisymmetric displacement caused by the same value of the EFCC current for the case $\phi_{0}=3 \pi / 4$. 
Studies presented in this work are focused on the effect of the interaction between sources of non-axisymmetric and axisymmetric external fields on the total plasma boundary displacement. Other type of interaction that could affect the value of the total plasma boundary displacement is the interaction of the non-axisymmetric external and non-axisymmetric plasma fields (so called plasma response effect). This type of interaction was not studied here (partly due to the relatively low values of $\beta_{\mathrm{N}}$ seen in the experiments used in this work) but it should be mentioned that similar type of analysis as is used here could be used also in order to study effect of the plasma response (as it was done for example in [6]).

\section{Conclusions}

3D plasma boundary displacement is studied in JET. Non-axisymmetric external magnetic field is applied with toroidal mode number $n=1$. Different amplitudes and several toroidal phase values are used. Studies are focused on the effect of the interaction between non-axisymmetric external field and shape control system on the total plasma displacement. Axisymmetric contribution to the total displacement is seen due to the direct pick-up of the non-axisymmetric field by the magnetic sensors used as input to the shape control system. It is shown that for the certain value of toroidal phase of the external field axisymmetric contribution can be avoided.

Direct measurements of the plasma boundary displacement at different toroidal locations are compared with the toroidal structure of the external filed for the two toroidal phase values. Nonaxisymmetic plasma boundary displacement is seen proportional to the main toroidal spectral component of the applied perturbation ( $n=1$ in our case). Plasma boundary displacement $\delta r \approx 3 \mathrm{~cm}$ is observed for the current value $\mathrm{I}_{\mathrm{EFCC}}=2 \mathrm{kA}$ in the EFCCs. It is found that axisymmetric contribution to the total displacement with similar value $(\delta \mathrm{r} \approx 3 \mathrm{~cm})$ can be caused by the interaction of the EFCCs and shape control system.

\section{Acknowledgments}

This work has been carried out within the framework of the EUROfusion Consortium and has received funding from the Euratom research and training programme 2014-2018 under grant agreement No 633053. The views and opinions expressed herein do not necessarily reflect those of the European Commission.

\section{References}

1. Chapman I.T. et al, 2007 Nucl. Fusion 47 L36

2. Yadykin. D. et al, 2014 Nucl. Fusion 54013016

3. Chapman I.T. et al, 2012 Plasma Phys. Control. Fusion 54105013

4. Fischer R. et al, 2012 Plasma Phys. Control. Fusion 54115008

5. Fuchs J.C. 2013 40th EPS Conf. of Plasma Physics (Helsinki, Finland) P4.126

(http://ocs.ciemat.es/EPS2013PAP/pdf/P4.126.pdf)

6. Lanctot M. J. et al, 2011 Phys. Plasmas 18056121 
7. Moyer R. A. et al, 2012 Nucl. Fusion 52123019

8. Ham C. J. 2014 Phys. Plasmas 21102501

9. Kirk A. et al, 2013 Nucl. Fusion $\mathbf{5 3} 043007$

10. Lazerson S. A. et al, $201239^{\text {th }}$ EPS Conf. on Plasma Physics (Stockholm, Sweden) http://epsicpp2012.spp.ee.kth.se/

11. Orain F. et al, 2013 Phys. Plasmas 20102510

12. Becoulet M. et al, $24^{\text {th }}$ IAEA Fusion Energy Conf. (San Diego, CA) TH/2-1 www. pub.iaea.org/iaeameetings/41985/24th-Fusion-Energy-Conference

13. Ferarro N.M. et al, 2012 Phys. Plasmas 19056105

14. Chapman I.T. et al, 2014 Nucl Fusion 54083006

15. Lister J.B., Portone A. and Gribov Y., 2006 IEEEControl Systems Magazine 2679

16. Gerasimov S. N. et al, 2014 Nucl. Fusion 54073009

17. Sartori F. et al, 2006 IEEE Control Systems Magazine 2664

18. Chapman I.T. et al, 2014 Plasma Phys. Control. Fusion 56, 075004

19. Paccagnella R., Gregoratto D. and Bondeson A ., 2002 Nucl. Fusion 421102

20. Yadikin D., Brunsell P.R and Drake J.R., 2006 Plasma Phys. Control. Fusion 481

21. Frassinetti L. et al, 2012 Rev. Sci. Instrum. 83013506 